\title{
On the Rate of Convergence for the Approximation of Nonlinear Problems
}

\author{
By J. Descloux, J. Rappaz and R. Scholz
}

\begin{abstract}
This paper shows how to obtain from estimates on linear problems error bounds in various norms for the approximation of nonlinear problems. The theory developed in this paper is applied to finite element methods for approximating the problem $-\Delta u=\lambda e^{u}$ and the Navier-Stokes equations.
\end{abstract}

1. Introduction. The aim of this paper is to present an abstract theory to obtain error estimates in various norms for the approximation of solution branches of nonlinear equations with the aid of known estimates for corresponding linear problems. We give a general analysis similar to that of Brezzi-Rappaz-Raviart [2] and apply it to the study of the convergence of finite element methods for nonlinear elliptic problems. Our analysis applies to regular points and simple limit points but not to bifurcation points.

In order to illustrate our results, we consider the following model problem. Let $\Omega$ be a bounded convex domain in $\mathbf{R}^{2}$ with sufficiently smooth boundary $\partial \Omega$. We are interested in approximating the solution of the boundary value problem

$$
-\Delta u=\lambda e^{u} \quad \text { in } \Omega, \quad u=0 \text { on } \partial \Omega,
$$

where $\lambda$ is a real parameter. It is well-known (see Amann [1], for instance) that there exists a maximum value $\lambda^{*}$ of the parameter $\lambda$ such that Problem (1.1) has at least one solution $u \in H_{0}^{1}(\Omega) \cap L^{\infty}(\Omega)$; moreover, there exists a unique solution $u^{*} \in$ $H_{0}^{1}(\Omega) \cap L^{\infty}(\Omega)$ of $(1.1)$ for $\lambda=\lambda^{*}$, and $\left(u^{*}, \lambda^{*}\right)$ is a turning point.

In order to parametrize the solution branch of Problem (1.1) which passes through $\left(u^{*}, \lambda^{*}\right)$, a new variable $t$ and a normalization equation are introduced as in Keller [8]. To this end we take a nontrivial function $\varphi^{*} \in H_{0}^{1}(\Omega)$ such that

$$
-\Delta \varphi^{*}=\lambda^{*} e^{u^{*}} \varphi^{*} \quad \text { in } \Omega,
$$

and we choose a continuous linear functional $\varphi$ on $L^{2}(\Omega)$ with $\varphi\left(\varphi^{*}\right) \neq 0$. Then there exist $t_{0}>0$ and a unique continuous mapping $t \in\left(-t_{0}, t_{0}\right) \rightarrow(u(t), \lambda(t)) \in$ $\left(H_{0}^{1}(\Omega) \cap L^{\infty}(\Omega)\right) \times \mathbf{R}$ such that for $|t|<t_{0}$ :

$$
\begin{gathered}
-\Delta u(t)=\lambda(t) e^{u(t)} \quad \text { in } \Omega \\
\varphi\left(u(t)-u^{*}\right)=t, \quad u(0)=u^{*}, \quad \lambda(0)=\lambda^{*} .
\end{gathered}
$$

Received August 22, 1983; revised June 25, 1984.

1980 Mathematics Subject Classification. Primary 65J15, 65N15, 65N30.

(1)1985 American Mathematical Society $0025-5718 / 85 \$ 1.00+\$ .25$ per page 
In order to compute an approximation of $\Gamma=\left\{(u(t), \lambda(t)):|t|<t_{0}\right\}$, we consider a finite element method for discretizing Problem (1.1). Let $V_{h}$ be the finite element subspace of $H_{0}^{1}(\Omega) \cap L^{\infty}(\Omega)$ of piecewise linear polynomials with respect to a triangulation of $\Omega$ with mesh size $h>0$. For the sake of simplicity we assume here that $\Omega$ is a polygonal domain; in the case of a curved boundary $\partial \Omega$ we use an appropriate modification of the functions of $V_{h}$ in the boundary triangles. An approximation $\left(u_{h}, \lambda\right) \in V_{h} \times \mathbf{R}$ of solutions of (1.1) is defined by

$$
D\left(u_{h}, v_{h}\right)=\lambda\left(e^{u_{h}}, v_{h}\right)_{0} \text { for all } v_{h} \in V_{h},
$$

where $D(\cdot, \cdot)$ and $(\cdot, \cdot)_{0}$ denote the Dirichlet integral and the $L^{2}(\Omega)$-scalar product, respectively.

Using the general results of Brezzi-Rappaz-Raviart [2], it is possible to prove that for $h \leqslant h_{0}$ and $\varepsilon>0$ small enough, there exists a unique continuous mapping $t \in\left(-t_{0}, t_{0}\right) \rightarrow\left(u_{h}(t), \lambda_{h}(t)\right) \in V_{h} \times \mathbf{R}$ such that for $|t|<t_{0}:$

$$
\begin{gathered}
D\left(u_{h}(t), v_{h}\right)=\lambda_{h}(t)\left(e^{u_{h}(t)}, v_{h}\right)_{0} \quad \forall v_{h} \in V_{h}, \\
\varphi\left(u_{h}(t)-u^{*}\right)=t, \quad\left\|u(t)-u_{h}(t)\right\|_{H^{1}(\Omega)}+\left|\lambda(t)-\lambda_{h}(t)\right|<\varepsilon .
\end{gathered}
$$

Moreover, error estimates for $\left|\lambda(t)-\lambda_{h}(t)\right|$ and $\left\|u(t)-u_{h}(t)\right\|_{H^{1}(\Omega)}$ are obtained; but by using this theory it is not possible to get optimal error estimates for $u(t)-u_{h}(t)$ in the $L^{2}$-norm or the $L^{\infty}$-norm, for example.

In Section 2 we give an abstract setting which permits us to obtain error estimates in various norms for the approximation of the solutions of nonlinear equations. In order to justify our formalism we return to the above example by setting $V=H_{0}^{1}(\Omega)$ $\cap L^{\infty}(\Omega), W=L^{2}(\Omega)$. If $T$ and $T_{h}$ are continuous linear operators from $W$ into $V$ defined, for $f \in W$, by

$$
T f=u \in V \quad \text { if } D(u, v)=(f, v)_{0} \text { for all } v \in V,
$$

and

$$
T_{h} f=u_{h} \in V_{h} \text { if } D\left(u_{h}, v_{h}\right)=\left(f, v_{h}\right)_{0} \text { for all } v_{h} \in V_{h},
$$

if $G: V \times \mathbf{R} \rightarrow W$ is the nonlinear mapping given by $G(u, \lambda)=-\lambda e^{u}$, then Problems (1.1) and (1.4) are, respectively, equivalent in finding pairs $(u, \lambda)$ and $\left(u_{h}, \lambda\right)$ in $V \times \mathbf{R}$ such that

$$
\begin{gathered}
F(u, \lambda):=u+T G(u, \lambda)=0, \\
F_{h}\left(u_{h}, \lambda\right):=u_{h}+T_{h} G\left(u_{h}, \lambda\right)=0 .
\end{gathered}
$$

We remark that if $\left(u_{h}, \lambda\right) \in V \times \mathbf{R}$ is a solution of (1.7), then $u_{h}$ belongs to the range of $T_{h}$ and we have $u_{h} \in V_{h}$. Result (1.5) is a direct consequence of the work of Brezzi-Rappaz-Raviart [2] and of the fact that $\lim _{h \rightarrow 0}\left\|T-T_{h}\right\|_{\mathscr{L}(W, V)}=0$. Moreover, we have the error estimate

$$
\left|\lambda(t)-\lambda_{h}(t)\right|+\left\|u(t)-u_{h}(t)\right\|_{V} \leqslant C\left\|F_{h}(\lambda(t), u(t))\right\|_{\nu},
$$

with $C$ independent of $h \leqslant h_{0}$ and $|t|<t_{0}$, which leads to an optimal error estimate $\left\|u(t)-u_{h}(t)\right\|_{H^{1}(\Omega)} \leqslant C h$. If we want to obtain optimal $L^{2}$-error estimates from the same theory, we would like to set Problems (1.6), (1.7) in $H \times \mathbf{R}$ instead of $V \times \mathbf{R}$, with $H=L^{2}(\Omega)$; unfortunately we cannot define $G$ on $H \times \mathbf{R}$, and, in Section 2, we develop an abstract setting for replacing $V$ by $H$ in the error estimate (1.8). 
In Section 3 we apply this theory to the above example and prove quasi-optimal $L^{2}$ - and $L^{\infty}$-estimates for the conforming as well as for a mixed finite element method. Section 4 is devoted to the conforming finite element method for the Navier-Stokes problem, using the "stream-function formulation" with optimal error estimates in the $H^{1}$-norm.

2. Abstract Error Estimates. In this section, $V$ and $W$ will represent real Banach spaces; $\mathscr{L}(W, V)$ is the space of bounded linear operators from $W$ to $V$. If there is no danger of confusion, the different norms $\|\cdot\|_{V},\|\cdot\|_{W}, \ldots$ will be denoted simply by $\|\cdot\|$. The norm of a product space of the form $V \times \mathbf{R}$ will be defined by $\|(v, \lambda)\|_{V \times \mathbf{R}}:=\|v\|_{V}+|\lambda|$.

Let $T$ and $T_{h}$ belong to $\mathscr{L}(W, V)$, and let $G: V \times \mathbf{R} \rightarrow W$ be a nonlinear $C^{p}$-mapping with $p \geqslant 2 ; h$ denotes a positive parameter, the values of which have an accumulation point at 0 .

In a neighborhood of a point $\left(u_{0}, \lambda_{0}\right) \in V \times \mathbf{R}$, we consider the nonlinear equations

$$
F(u, \lambda)=0, \quad F_{h}(u, \lambda)=0,
$$

where $F$ and $F_{h}: V \times \mathbf{R} \rightarrow V$ are nonlinear mappings defined by

$$
F(u, \lambda)=u+T G(u, \lambda), \quad F_{h}(u, \lambda)=u+T_{h} G(u, \lambda) .
$$

For an intuitive meaning of $V, W, G, T, T_{h}, F$, and $F_{h}$, the reader may refer to the example of Section 1.

We first suppose:

(a) $F\left(u_{0}, \lambda_{0}\right)=0$,

(b) $T$ is a compact operator,

(c) $\lim _{h \rightarrow 0}\left\|T-T_{h}\right\|_{\mathscr{L}(W, V)}=0$.

Denoting by $F^{\prime}(u, \lambda) \in \mathscr{L}(V \times \mathbf{R}, V)$, by $D_{u} F(u, \lambda) \in \mathscr{L}(V, V)$, and by $D_{\lambda} F(u, \lambda)$ $\in \mathscr{L}(\mathbf{R}, V)$, respectively, the total derivative of $F$ at $(u, \lambda)$ and the partial derivatives of $F$ with respect to $u$ and $\lambda$, we remark that Hypothesis (2.4) implies that $D_{u} F(u, \lambda)$ is a Fredholm operator of index 0 and, consequently, that $F^{\prime}(u, \lambda)$ is a Fredholm operator of index 1.

We next suppose either that $D_{u} F\left(u_{0}, \lambda_{0}\right)$ is an isomorphism from $V$ into itself, or that $D_{u} F\left(u_{0}, \lambda_{0}\right)$ has a kernel of dimension 1 and $D_{\lambda}\left(u_{0}, \lambda_{0}\right)$ does not belong to the range of $D_{u} F\left(u_{0}, \lambda_{0}\right)$; in the first case, $\left(u_{0}, \lambda_{0}\right)$ is a "regular point"; in the second case, $\left(u_{0}, \lambda_{0}\right)$ is a "simple limit point". This assumption can be written simply as

(d) Range $F^{\prime}\left(u_{0}, \lambda_{0}\right)=V$.

The following result can be found in Descloux-Rappaz [4].

THEOREM 2.1. Under Hypotheses (2.1)-(2.6), there exist $h_{0}>0$ and a neighborhood of $\left(u_{0}, \lambda_{0}\right) \in V \times \mathbf{R}$ such that, for $h \leqslant h_{0}$, and in this neighborhood, each of the equations $F(u, \lambda)=0$ and $F_{h}(u, \lambda)=0$ possesses a unique branch of solutions. These branches can be parametrized as $(u(t), \lambda(t)),\left(u_{h}(t), \lambda_{h}(t)\right),|t| \leqslant t_{0}, t_{0}>0$, with the following properties:

( $\alpha)(u(t), \lambda(t))$ and $\left(u_{h}(t), \lambda_{h}(t)\right)$ are of class $C^{p} ;(u(0), \lambda(0))=\left(u_{0}, \lambda_{0}\right)$; $u^{\prime}(0) \neq 0$ 
(B) $\lim _{h \rightarrow 0} \sup _{|t| \leqslant t_{0}}\left\{\left\|u^{(k)}(t)-u_{h}^{(k)}(t)\right\|_{V}+\left|\lambda^{(k)}(t)-\lambda_{h}^{(k)}(t)\right|\right\}=0, \quad 0 \leqslant k \leqslant$ $p-1$, where $u^{(k)}, \lambda^{(k)}, \ldots$ are the kth derivatives of $u, \lambda, \ldots$ with respect to $t$;

$(\gamma)$ there exists a constant $C$ such that, for $|t| \leqslant t_{0}, h \leqslant h_{0}, 0 \leqslant k \leqslant p-1$, we have

$$
\left\|u^{(k)}(t)-u_{h}^{(k)}(t)\right\|_{V}+\left|\lambda^{(k)}(t)-\lambda_{h}^{(k)}(t)\right| \leqslant C \sum_{l=0}^{k}\left\|\frac{d^{l}}{d t^{l}} F_{h}(u(t), \lambda(t))\right\|_{V} .
$$

The purpose of this section is to derive error estimates for $u^{(k)}(t)-u_{h}^{(k)}(t)$ in a norm different from $\|\cdot\|_{v}$. Let $H$ be a Banach space for which we suppose

(e) $V \subset H$ with continuous injection;

(f) there exists a constant $C$ such that along the solution branch of the exact problem $(u(t), \lambda(t))$ defined by Theorem 2.1 , we have

$$
\left\|D_{\lambda}^{k-l} D_{u}^{l} G(u(t), \lambda(t))\left[v_{1}, \ldots, v_{l}\right]\right\|_{W} \leqslant C\left\|v_{1}\right\|_{H} \prod_{i=1}^{l-1}\left\|v_{i}\right\|_{V}
$$

for $|t| \leqslant t_{0}, 1 \leqslant k \leqslant p-1,1 \leqslant l \leqslant k$, and for all $v_{1}, \ldots, v_{l} \in V$.

Our main abstract result is contained in the following

TheOREM 2.2. We assume that Hypotheses (2.1)-(2.8) are satisfied. Then, for the exact and the approximate branches of solutions defined by Theorem 2.1, there exist constants $t_{0}, h_{0}$, and $C$ and parametrizations $(u(t), \lambda(t)),\left(u_{h}(t), \lambda_{h}(t)\right)$, such that, for $0 \leqslant k \leqslant p-2,|t| \leqslant t_{0}, h \leqslant h_{0}$, we have

$$
\begin{aligned}
& \left\|u^{(k)}(t)-u_{h}^{(k)}(t)\right\|_{H}+\left|\lambda^{(k)}(t)-\lambda_{h}^{(k)}(t)\right| \\
& \leqslant C\left\{\sum_{l=0}^{k}\left\|\frac{d^{\prime}}{d t^{\prime}} F_{h}(u(t), \lambda(t))\right\|_{H}+\left\|F_{h}(u(t), \lambda(t))\right\|_{V}^{2}\right\} .
\end{aligned}
$$

Remark 2.1. In Theorems 2.1 and 2.2, the parametrizations of the exact (respectively, of the approximate) solution branch are not necessarily identical; however, in the proofs we shall show that it is possible to choose the same ones.

Remark 2.2. In general, Theorem 2.2 gives better bounds for $\left|\lambda^{(k)}(t)-\lambda_{h}^{(k)}(t)\right|$ than Theorem 2.1 does.

Remark 2.3. In many examples, Hypothesis (2.8) will be verified by using regularity properties of the solutions of the exact problem.

Remark 2.4. Hypotheses (2.4) and (2.5) can be weakened by using results of Descloux-Rappaz [4].

We first prove the following auxiliary result.

Lemma 2.1. Let $R \in \mathscr{L}(W, V), S \in \mathscr{L}(V, W), a \in W, \alpha \in \mathbf{R}, \varphi \in \mathscr{L}(V, \mathbf{R})$; let $A \in \mathscr{L}(V \times \mathbf{R}, V \times \mathbf{R})$ and $B \in \mathscr{L}(W \times \mathbf{R}, W \times \mathbf{R})$ be the linear operators defined by

$$
\begin{aligned}
& A(v, \lambda)=(v+R S v+\lambda R a, \varphi(v)+\alpha \lambda), \\
& B(w, \lambda)=(w+S R w+\lambda a, \varphi(R w)+\alpha \lambda) .
\end{aligned}
$$

We suppose that $A$ is an isomorphism. Then $B$ is an isomorphism and

$$
\left\|B^{-1}\right\| \leqslant 1+(1+\|R\|)(1+\|S\|+\|a\|)\left\|A^{-1}\right\| .
$$

Proof. We only verify the surjectivity of $B$. Let $g \in W, \gamma \in \mathbf{R}$ be given; setting $(v, \lambda)=A^{-1}(R g, \gamma), w=g-\lambda a-S v$, if follows that $B(w, \lambda)=(g, \gamma)$. From the expressions of $w$ and $\lambda$, one deduces easily the bound for $\left\|B^{-1}\right\|$. 
In the following, we shall suppose that Hypotheses (2.3)-(2.8) are satisfied. By (2.6), since $F^{\prime}\left(u_{0}, \lambda_{0}\right)$ is a Fredholm operator of index 1 , it follows that the kernel of $F^{\prime}\left(u_{0}, \lambda_{0}\right)$ has dimension 1. Let $(z, \omega) \in V \times \mathbf{R}$ be a nonvanishing element of the kernel of $F^{\prime}\left(u_{0}, \lambda_{0}\right)$. By (2.7) and the Hahn-Banach theorem, there exist $\varphi \in \mathscr{L}(H, \mathbf{R})$ and $\alpha \in \mathbf{R}$ such that

$$
\varphi(z)+\alpha \omega \neq 0 .
$$

Let us define the mappings $\mathscr{F}: V \times \mathbf{R} \times \mathbf{R} \rightarrow V \times \mathbf{R}$ and $\mathscr{G}: W \times \mathbf{R} \times \mathbf{R} \rightarrow W \times \mathbf{R}$ by

$$
\begin{aligned}
& \mathscr{F}(u, \lambda, t)=\left(u+T G(u, \lambda), \varphi\left(u-u_{0}\right)+\alpha\left(\lambda-\lambda_{0}\right)-t\right), \\
& \mathscr{G}(w, \lambda, t)=\left(w+G(T w, \lambda), \varphi\left(T w-u_{0}\right)+\alpha\left(\lambda-\lambda_{0}\right)-t\right) .
\end{aligned}
$$

Furthermore, we set $w_{0}=-G\left(u_{0}, \lambda_{0}\right)$ and notice that, by (2.3), we have $u_{0}=T w_{0}$ and, consequently, $\mathscr{F}\left(u_{0}, \lambda_{0}, 0\right)=0, \mathscr{G}\left(w_{0}, \lambda_{0}, 0\right)=0$. (2.8) and (2.9) imply that $D_{(u, \lambda)} \mathscr{F}\left(u_{0}, \lambda_{0}, 0\right) \in \mathscr{L}(V \times \mathbf{R}, V \times \mathbf{R})$ is an isomorphism; setting, in Lemma 2.1, $R=T, S=D_{u} G\left(u_{0}, \lambda_{0}\right), a=D_{\lambda} G\left(u_{0}, \lambda_{0}\right)$, we see that $A=D_{(u, \lambda)} \mathscr{F}\left(u_{0}, \lambda_{0}, 0\right)$ and that $B=D_{(w, \lambda)} \mathscr{G}\left(w_{0}, \lambda_{0}, 0\right) \in \mathscr{L}(W \times \mathbf{R}, W \times \mathbf{R})$ is an isomorphism. We next define $\mathscr{F}_{h}: V \times \mathbf{R} \times \mathbf{R} \rightarrow V \times \mathbf{R}$ and $\mathscr{G}_{h}: W \times \mathbf{R} \times \mathbf{R} \rightarrow W \times \mathbf{R}$ by the right-hand members of (2.10) and (2.11) when $T$ is replaced by $T_{h}$. By (2.5) there exists a neighborhood $\mathscr{V}$ of $\left(u_{0}, \lambda_{0}, 0\right) \in V \times \mathbf{R} \times \mathbf{R}$ and a neighborhood $\mathscr{W}$ of $\left(w_{0}, \lambda_{0}, 0\right)$ $\in W \times \mathbf{R} \times \mathbf{R}$ such that, for $0 \leqslant k \leqslant p-1, \lim _{h \rightarrow 0} \mathscr{F}_{h}^{(k)}=\mathscr{F}^{(k)}$ uniformly in $\mathscr{V}$ and $\lim _{h \rightarrow 0} \mathscr{G}_{h}^{(k)}=\mathscr{G}^{(k)}$ uniformly in $\mathscr{W}$. By the classical implicit function theorem applied to $\mathscr{F}$ and $\mathscr{G}$ and by its special version given in Brezzi-Rappaz-Raviart [2] applied to $\mathscr{F}_{h}$ and $\mathscr{G}_{h}$, we obtain

LEMMA 2.2. ( $\alpha$ ) There exist positive constants $t_{0}, h_{0}$, and $C_{0}$, and for $|t| \leqslant t_{0}, h \leqslant h_{0}$ there exist unique elements $(u(t), \lambda(t)) \in V \times \mathbf{R}, \quad(w(t), \mu(t)) \in W \times \mathbf{R}$, $\left(u_{h}(t), \lambda_{h}(t)\right) \in V \times \mathbf{R},\left(w_{h}(t), \mu_{h}(t)\right) \in W \times \mathbf{R}$ such that

$$
\begin{array}{ll}
\mathscr{F}(u(t), \lambda(t), t)=0, & \left\|u(t)-u_{0}\right\|_{V}+\left|\lambda(t)-\lambda_{0}\right| \leqslant C_{0}, \\
\mathscr{G}(w(t), \mu(t), t)=0, & \left\|w(t)-w_{0}\right\|_{W}+\left|\mu(t)-\lambda_{0}\right| \leqslant C_{0}, \\
\mathscr{F}_{h}\left(u_{h}(t), \lambda_{h}(t), t\right)=0, & \left\|u_{h}(t)-u_{0}\right\|_{V}+\left|\lambda_{h}(t)-\lambda_{0}\right| \leqslant C_{0}, \\
\mathscr{G}_{h}\left(w_{h}(t), \mu_{h}(t), t\right)=0, & \left\|w_{h}(t)-w_{0}\right\|_{W}+\left|\mu_{h}(t)-\lambda_{0}\right| \leqslant C_{0} ;
\end{array}
$$

moreover, all functions $u(t), \lambda(t), w(t), \mu(t), \ldots$ are $C^{p}$-mappings.

( $\beta$ ) For $0 \leqslant k \leqslant p-1$ we have

$$
\begin{aligned}
\lim _{h \rightarrow 0} \sup _{|t| \leqslant t_{0}}\{ & \left\|u^{(k)}(t)-u_{h}^{(k)}(t)\right\|_{V}+\left|\lambda^{(k)}(t)-\lambda_{h}^{(k)}(t)\right| \\
& \left.+\left\|w^{(k)}(t)-w_{h}^{(k)}(t)\right\|_{w}+\left|\mu^{(k)}(t)-\mu_{h}^{(k)}(t)\right|\right\}=0 .
\end{aligned}
$$

( $\gamma$ ) There exists a constant $C$ such that, for $0 \leqslant k \leqslant p-1,|t| \leqslant t_{0}$, and $h \leqslant h_{0}$, we have

$$
\begin{aligned}
& \left\|u^{(k)}(t)-u_{h}^{(k)}(t)\right\|_{V}+\left|\lambda^{(k)}(t)-\lambda_{h}^{(k)}(t)\right| \leqslant C \sum_{l=0}^{k}\left\|\frac{d^{l}}{d t^{l}} \mathscr{F}_{h}(u(t), \lambda(t), t)\right\|_{V \times \mathbf{R}} \\
& \left\|w^{(k)}(t)-w_{h}^{(k)}(t)\right\|_{W}+\left|\mu^{(k)}(t)-\mu_{h}^{(k)}(t)\right| \leqslant C \sum_{l=0}^{k}\left\|\frac{d^{l}}{d t^{l}} \mathscr{G}_{h}(w(t), \mu(t), t)\right\|_{W \times \mathbf{R}} .
\end{aligned}
$$


Note that, in Lemma 2.2, $t$ is a pseudo-arclength parameter, as in Keller [8]. Clearly Theorem 2.1 is a direct consequence of Lemma 2.2, and we now proceed to the

Proof of Theorem 2.2. We remark that $\mathscr{G}(-G(u(t), \lambda(t)), \lambda(t), t)=0$ and $\mathscr{G}_{h}\left(-G\left(u_{h}(t), \lambda_{h}(t)\right), \lambda_{h}(t), t\right)=0$ for $|t| \leqslant t_{0}$; by the uniqueness of the quantities $u(t), \lambda(t), \ldots$ in Lemma 2.2, we deduce the following key relations:

$$
\left\{\begin{array}{lc}
w(t)=-G(u(t), \lambda(t)), \quad u(t)=T w(t), \\
w_{h}(t)=-G_{h}\left(u_{h}(t), \lambda_{h}(t)\right), \quad u_{h}(t)=T_{h} w_{h}(t), \\
\mu(t)=\lambda(t), \mu_{h}(t)=\lambda_{h}(t) \quad \text { for }|t| \leqslant t_{0}, h \leqslant h_{0}
\end{array}\right.
$$

In the following, $C$ will denote a generic constant.

Since $G$ is a map of class $C^{p}$, we can assume, without loss of generality, that for $|t| \leqslant t_{0}, h \leqslant h_{0},(T w(t), \lambda(t))=(u(t), \lambda(t))$, and $\left(T_{h} w(t), \lambda(t)\right)$ belong to a convex neighborhood $\mathscr{V} \subset V \times \mathbf{R}$ of $\left(u_{0}, \lambda_{0}\right)$ for which we have

$$
\sup _{(v, \lambda) \in \mathscr{V}}\left\|G^{(k)}(v, \lambda)\right\| \leqslant C, \quad 0 \leqslant k \leqslant p,
$$

where $\|\cdot\|$ represents the norm of the $k$-multilinear operators from $(V \times \mathbf{R})^{k}$ into $W$.

Since $\mathscr{G}(w(t), \mu(t), t)=0$, we obtain, by Lemma 2.2 and (2.12) for $0 \leqslant k \leqslant p-1$, $|t| \leqslant t_{0}, h \leqslant h_{0}$ :

$$
\begin{aligned}
\left\|w^{(k)}(t)-w_{h}^{(k)}(t)\right\|_{W}+\left|\lambda^{(k)}(t)-\lambda_{h}^{(k)}(t)\right| & \\
\leqslant C \sum_{l=0}^{k}\left\{\| \frac{d^{l}}{d t^{\prime}}\left(G\left(T_{h} w(t), \lambda(t)\right)-\right.\right. & G(T w(t), \lambda(t))) \|_{W} \\
& \left.+\left\|\left(T_{h}-T\right) w^{(l)}(t)\right\|_{H}\right\},
\end{aligned}
$$

where we have used Hypothesis (2.7) and the fact that $\varphi \in \mathscr{L}(H, \mathbf{R})$. Furthermore, by (2.7), (2.12) and the uniform boundedness of the operators $T_{h} \in \mathscr{L}(W, V)$, we have

$$
\begin{aligned}
\left\|u^{(k)}(t)-u_{h}^{(k)}(t)\right\|_{H} & \leqslant\left\|\left(T_{h}-T\right) w^{(k)}(t)\right\|_{H}+\left\|T_{h}\left(w^{(k)}(t)-w_{h}^{(k)}(t)\right)\right\|_{H} \\
& \leqslant\left\|\left(T_{h}-T\right) w^{(k)}(t)\right\|_{H}+C\left\|w^{(k)}(t)-w_{h}^{(k)}(t)\right\|_{W} .
\end{aligned}
$$

Clearly, the relation $\mathscr{F}(u(t), \lambda(t), t)=0$ implies $F(u(t), \lambda(t))=0$ (see (2.2)), and by (2.12) we have $F_{h}(u(t), \lambda(t))=\left(T-T_{h}\right) w(t)$; consequently, by (2.14) and (2.15), the proof of Theorem 2.2 will be completed if we establish that, for $0 \leqslant k \leqslant p-2,|t| \leqslant t_{0}$, and $h \leqslant h_{0}$, we have

$$
\begin{aligned}
& \sum_{l=0}^{k}\left\|\frac{d^{l}}{d t^{\prime}}\left(G\left(T_{h} w(t), \lambda(t)\right)-G(T w(t), \lambda(t))\right)\right\|_{W} \\
& \quad \leqslant C\left\{\sum_{l=0}^{k}\left\|\left(T_{h}-T\right) w^{(l)}(t)\right\|_{H}+\left\|\left(T_{h}-T\right) w(t)\right\|_{V}^{2}\right\} .
\end{aligned}
$$

For the sake of simplicity, we verify (2.16) only for $k=0$ and $k=1$. We shall use the notations $\underline{V}=V \times \mathbf{R}, \underline{H}=H \times \mathbf{R}, G(\underline{v})=G(v, \lambda)$ for $\underline{v}=(v, \lambda) \in \underline{V}$, and we 
set $\underline{u}(t)=(u(t), \lambda(t))=(T w(t), \lambda(t)), \underline{v}_{h}(t)=\left(T_{h} w(t), \lambda(t)\right)$. For $k=1, k=2$, Hypothesis (2.8) implies

$$
\begin{gathered}
\left\|G^{\prime}(\underline{u}(t)) \underline{v}\right\|_{W} \leqslant C\|\underline{v}\|_{\underline{H}}, \\
\left\|G^{\prime \prime}(\underline{u}(t))\left[\underline{v}_{1}, \underline{v}_{2}\right]\right\|_{W} \leqslant C\left\|\underline{v}_{1}\right\|_{\underline{V}}\left\|_{\underline{v}_{2}}\right\|_{\underline{H}}
\end{gathered}
$$

for all $\underline{v}, \underline{v}_{1}, \underline{v}_{2} \in \underline{V}$ and all $|t| \leqslant t_{0}$. By (2.13), (2.17), and Taylor's formula, we get

$$
\left\|G\left(\underline{v}_{h}\right)-G(\underline{u})\right\|_{W} \leqslant C\left\{\left\|\underline{v}_{h}-\underline{u}_{\underline{H}}+\right\| \underline{v}_{h}-\underline{u} \|_{\underline{\underline{v}}}^{2}\right\},
$$

where, from now on, we simply write $\underline{u}^{(k)}$ and $\underline{v}_{h}^{(k)}$ for $\underline{u}^{(k)}(t)$ and $\underline{v}_{h}^{(k)}(t)$. (2.18) proves (2.16) for $k=0$. With the same arguments we obtain

$$
\begin{aligned}
\| \frac{d}{d t} & \left(G\left(\underline{v}_{h}\right)-G(\underline{u})\right)\left\|_{W}=\right\| G^{\prime}\left(\underline{v}_{h}\right) \underline{v}_{h}^{\prime}-G^{\prime}\left(\underline{u}_{)} \underline{u}^{\prime} \|_{W}\right. \\
& \leqslant\left\|\left(G^{\prime}\left(\underline{v}_{h}\right)-G^{\prime}(\underline{u})\right) \underline{v}_{h}^{\prime}\right\|_{W}+\left\|G^{\prime}\left(\underline{u}^{\prime}\right)\left(\underline{v}_{h}^{\prime}-\underline{u}^{\prime}\right)\right\|_{W} \\
& \leqslant\left\|G^{\prime \prime}\left(\underline{u}^{\prime}\right)\left[\underline{v}_{h}^{\prime}, \underline{v}_{h}-\underline{u}\right]\right\|_{W}+C\left\|\underline{\underline{v}}_{h}^{\prime}\right\|_{\underline{\underline{y}}}\left\|\underline{v}_{h}-\underline{u}\right\|_{\underline{\underline{V}}}^{2}+\left\|G^{\prime}\left(\underline{u}^{\prime}\right)\left(\underline{v}_{h}^{\prime}-\underline{u}^{\prime}\right)\right\|_{W} \\
& \leqslant C\left\{\left\|\underline{v}_{h}-\underline{u}\right\|_{\underline{H}}+\left\|\underline{v}_{h}^{\prime}-\underline{u}^{\prime}\right\|_{\underline{H}}+\left\|\underline{v}_{h}-\underline{u}\right\|_{\underline{\underline{V}}}^{2}\right\}
\end{aligned}
$$

where we have used the boundedness of $\left\|\underline{v}_{h}^{\prime}\right\|_{\underline{V}}$ and relation (2.13) for $k=1,2,3$; (2.19) proves relation (2.16) for $k=1$.

3. Application I: A Second-Order Boundary Value Problem. Let $\Omega \subset \mathbf{R}^{2}$ be a bounded convex domain with sufficiently smooth boundary $\partial \Omega$. We consider the nonlinear boundary value problem

$$
-\Delta u=\lambda e^{u} \quad \text { in } \Omega, \quad u=0 \quad \text { on } \partial \Omega .
$$

We will show that the abstract results can be applied in the context of approximation of branches of solutions of (3.1) by conforming and nonconforming finite element methods.

First we will consider the case of conforming finite elements. We use the standard notation of Sobolev spaces $H_{0}^{1}(\Omega), H^{m}(\Omega), W^{m, p}(\Omega), \ldots$ and set $V:=H_{0}^{1}(\Omega) \cap$ $L^{\infty}(\Omega), W:=L^{2}(\Omega)$. A weak formulation of (3.1) is

Find $(u, \lambda) \in V \times \mathbf{R}$ with

$$
D(u, v)=\lambda\left(e^{u}, v\right)_{0} \text { for all } v \in V,
$$

where $D(\cdot, \cdot)$ and $(\cdot, \cdot)_{0}$ denote Dirichlet's integral and the $L^{2}(\Omega)$-scalar product, respectively.

We define the operator $T \in \mathscr{L}(W, V)$ by

$$
T f=u \quad \text { if } D(u, v)=(f, v)_{0} \text { for all } v \in V
$$

holds, and the nonlinear operator $G: V \times \mathbf{R} \rightarrow W$ is given by $G(u, \lambda):=-\lambda e^{u}$. Then (3.2) is equivalent to the following problem:

Find $(u, \lambda) \in V \times \mathbf{R}$ with

$$
F(u, \lambda):=u+T G(u, \lambda)=0 .
$$

To determine approximate solutions of (3.3) we consider subspaces $V_{h} \subset V$, which consist of continuous, piecewise linear functions with respect to regular triangulations of $\Omega$ with mesh-size $h>0$. (In the case of a curved boundary $\partial \Omega$ we use an appropriate modification of the functions of $V_{h}$ in the boundary triangles. See 
Zlamal [16], for example.) With respect to the approximation properties of the spaces $V_{h}$ we refer to Ciarlet [3], for example. We define $T_{h} \in \mathscr{L}\left(W, V_{h}\right)$ by

$$
T_{h} f=u_{h} \text { if } D\left(u_{h}, \varphi\right)=(f, \varphi)_{0} \text { for all } \varphi \in V_{h} .
$$

The following estimates are known:

$$
\begin{gathered}
\left\|\left(T-T_{h}\right) f\right\|_{L^{2}(\Omega)}+h\left\|\left(T-T_{h}\right) f\right\|_{H_{0}^{1}(\Omega)} \leqslant C h^{2}\|f\|_{L^{2}(\Omega)} \\
\left\|\left(T-T_{h}\right) f\right\|_{L^{\infty}(\Omega)} \leqslant C h\|f\|_{L^{2}(\Omega)}
\end{gathered}
$$

Here $C$ denotes numerical constants which are independent of $f$ and $h$. For proofs of (3.4) and (3.6) we refer to Nitsche [10], [11]. Estimate (3.5) follows from (3.4) and inverse estimates. An immediate consequence of (3.4) and (3.5) is

$$
\left\|T-T_{h}\right\|_{\mathscr{L}(w, V)} \leqslant C h .
$$

The Galerkin problem to approximate the solution of (3.3) is formulated as follows:

Find $\left(u_{h}, \lambda\right) \in V \times \mathbf{R}$ with

$$
F_{h}\left(u_{h}, \lambda\right):=u_{h}+T_{h} G\left(u_{h}, \lambda\right)=0 .
$$

Note that if $\left(u_{h}, \lambda\right)$ is a solution of (3.8), then $u_{h}$ belongs to the range of $T_{h}$ and we have $u_{h} \in V_{h}$.

Assume $\left(u_{0}, \lambda_{0}\right) \in V \times \mathbf{R}$ fulfills (2.3) and (2.6). First of all, by Theorem 2.1, we have:

For $|t| \leqslant t_{0}, h \leqslant h_{0}$, there exist branches $(u(t), \lambda(t)),\left(u_{h}(t), \lambda_{h}(t)\right)$ of solutions of (3.3) and (3.8), respectively, with $(u(0), \lambda(0))=\left(u_{0}, \lambda_{0}\right)$ such that, for $k \in \mathbf{N}$,

$$
\left\|u^{(k)}(t)-u_{h}^{(k)}(t)\right\|_{H_{0}^{1}(\Omega) \cap L^{\infty}(\Omega)}+\left|\lambda^{(k)}(t)-\lambda_{h}^{(k)}(t)\right| \leqslant C_{k} h
$$

holds, where $C_{k}$ is independent of $h$ and $t$.

The proof of (3.9) is an immediate consequence of (3.7) and the fact that $G$ is of class $C^{\infty}$. Applying Theorem 2.2, we get

THEOREM 3.1. For the branches $(u(t), \lambda(t)),\left(u_{h}(t), \lambda_{h}(t)\right)$ as above, the estimate

$$
\left\|u^{(k)}(t)-u_{h}^{(k)}(t)\right\|_{L^{2}(\Omega)}+\left|\lambda^{(k)}(t)-\lambda_{h}^{(k)}(t)\right| \leqslant C_{k} h^{2}
$$

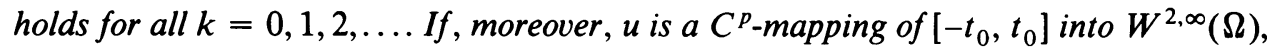
$p \geqslant 2$, then, for $0 \leqslant k \leqslant p-2$,

$$
\left\|u^{(k)}(t)-u_{h}^{(k)}(t)\right\|_{L^{\infty}(\Omega)} \leqslant C_{k} h^{2}|\ln h|
$$

holds. Here $C_{k}$ denotes constants which are independent of $t$ and $h$.

Proof. First we set $H:=L^{2}(\Omega)$. Then Assumption (2.7) is trivial; to apply Theorem 2.2 we have to prove (2.8). Since we can write $G(u, \lambda)=\lambda G(u)$ with $G(u)=e^{u}$, we only have to show

$$
\left\|D^{\prime} G(u)\left[v_{1}, \ldots, v_{l}\right]\right\|_{W} \leqslant C\left\|v_{l}\right\|_{H} \prod_{i=1}^{l-1}\left\|v_{i}\right\|_{V}
$$

with $W=L^{2}(\Omega), \quad V=H_{0}^{1}(\Omega) \cap L^{\infty}(\Omega)$. Since $D^{\prime} G(u)\left[v_{1}, \ldots, v_{l}\right]=e^{u} \cdot v_{1} \cdots v_{l}$ holds, (3.12) follows with

$$
C=\exp \left(\|u\|_{L^{\infty}(\Omega)}\right)
$$


Now (3.10) is a direct consequence of Theorem 2.2 and estimate (3.4). To show (3.11) we set $H:=L^{\infty}(\Omega)$. Then (2.7) and (2.8) are trivial and the result follows by combining Theorem (2.2) and estimates (3.4) and (3.6).

Next we will apply a mixed finite element method to get approximations of a branch of solutions of Problem (3.1). In this case we set $V:=L^{\infty}(\Omega) \times L^{2}(\Omega)^{2}$ and $W:=L^{2}(\Omega)$. Furthermore, let $H(\operatorname{div} ; \Omega)$ be the subspace of those elements $z \in$ $L^{2}(\Omega)^{2}$ with $\operatorname{div} z \in L^{2}(\Omega)$. Using these notations, another weak formulation of (3.1) is:

Find $\left(\left(u_{1}, u_{2}\right), \lambda\right) \in V \times \mathbf{R}$ with

$$
\begin{array}{ll}
-\left(u_{1}, \operatorname{div} z\right)_{0}=\left(u_{2}, z\right)_{0} & \text { for all } z \in H(\operatorname{div} ; \Omega), \\
-\left(\operatorname{div} u_{2}, w\right)_{0}=\lambda\left(e^{u_{1}}, w\right)_{0} & \text { for all } w \in L^{2}(\Omega),
\end{array}
$$

where $(\cdot, \cdot)_{0}$ denotes the scalar product in $L^{2}(\Omega)$ as well as in $L^{2}(\Omega)^{2}$.

In this case the operator $T: W \rightarrow V$ is defined as follows. For $f \in W$ denote by $u=\left(u_{1}, u_{2}\right) \in L^{2}(\Omega) \times H(\operatorname{div} ; \Omega)$ the solution of

$$
\begin{array}{ll}
-\left(u_{1}, \operatorname{div} z\right)_{0}=\left(u_{2}, z\right)_{0} & \text { for all } z \in H(\operatorname{div} ; \Omega), \\
-\left(\operatorname{div} u_{2}, w\right)_{0}=(f, w)_{0} & \text { for all } w \in L^{2}(\Omega) .
\end{array}
$$

Using shift theorems and Sobolev's imbedding theorem, we get $T f=u \in V$.

Further, let $G: V \times \mathbf{R} \rightarrow W$ be given by $G(u, \lambda):=-\lambda e^{u_{1}}$. Then Problem (3.13) is equivalent to:

Find $(u, \lambda) \in V \times \mathbf{R}$ with

$$
F(u, \lambda):=u+T G(u, \lambda)=0 .
$$

To determine approximations of the solution of (3.14) by a mixed finite element method, we use the subspaces introduced by Raviart-Thomas [12]. With respect to a quasi-uniform triangulation of $\Omega$ with mesh size $h>0$, we denote by $S_{h}$ the space of piecewise linear functions without any boundary conditions on $\partial \Omega$ or continuity requirements across interelement boundaries. $H_{h}$ are subspaces of $H(\operatorname{div} ; \Omega)$ which consist of pairs $\zeta=\left(\zeta_{1}, \zeta_{2}\right)$ such that $\zeta_{1}$ and $\zeta_{2}$ are special polynomials of degree 2 . We set $V_{h}:=S_{h} \times H_{h}$; the operator $T_{h}: W \rightarrow V_{h}$ is defined by $T_{h} f=u_{h}=\left(u_{1, h}, u_{2, h}\right)$ if

$$
\begin{array}{ll}
-\left(u_{1, h}, \operatorname{div} \zeta\right)_{0}=\left(u_{2, h}, \zeta\right)_{0} & \text { for all } \zeta \in H_{h}, \\
-\left(\operatorname{div} u_{2, h}, \omega\right)_{0}=(f, \omega)_{0} & \text { for all } \omega \in S_{h} .
\end{array}
$$

The following estimates for $u=T f$ and $u_{h}=T_{h} f$ are known:

$$
\begin{gathered}
\left\|u_{1}-u_{1, h}\right\|_{L^{2}(\Omega)}+h\left\|u_{2}-u_{2, h}\right\|_{L^{2}(\Omega)^{2}} \leqslant C h^{2}\|f\|_{L^{2}(\Omega)}, \\
\left\|u_{1}-u_{1, h}\right\|_{L^{\infty}(\Omega)} \leqslant C h\|f\|_{L^{2}(\Omega)} .
\end{gathered}
$$

For a proof of (3.15) see Raviart-Thomas [12], Scholz [13]; (3.16) follows from (3.15) and inverse estimates. A direct consequence of (3.15) and (3.16) is

$$
\left\|T-T_{h}\right\|_{\mathscr{L}(W, V)} \leqslant C h .
$$

The Galerkin problem corresponding to (3.14) is defined by

$$
F_{h}\left(u_{h}, \lambda\right):=u_{h}+T_{h} G\left(u_{h}, \lambda\right)=0 .
$$


If (2.3) and (2.6) are satisfied, for $|t| \leqslant t_{0}, h \leqslant h_{0}, k \in N$, according to Theorem 2.1 there exist branches $\left(\left(u_{1}(t), u_{2}(t)\right), \lambda(t)\right)$ and $\left(\left(u_{1, h}(t), u_{2, h}(t)\right), \lambda_{h}(t)\right)$ with

$$
\begin{aligned}
\left\|u_{1}^{(k)}(t)-u_{1, h}^{(k)}(t)\right\|_{L^{\infty}(\Omega)}+\left\|u_{2}^{(k)}(t)-u_{2, h}^{(k)}(t)\right\|_{L^{2}(\Omega)^{2}} & \\
& +\left|\lambda^{(k)}(t)-. \lambda_{h}^{(k)}(t)\right| \leqslant C_{k} h,
\end{aligned}
$$

where $C_{k}$ is independent of $t$ and $h$.

To get a better order of convergence for the approximation of $u_{1}$ in the $L^{2}(\Omega)$-norm, we need

LEMMA 3.1. Let $f \in W$ be given and set $u=\left(u_{1}, u_{2}\right)=T f$ and $u_{h}=\left(u_{1, h}, u_{2, h}\right)=$ $T_{h} f$. Then the estimate

$$
\left\|u_{2}-u_{2, h}\right\|_{H^{-1}(\Omega)^{2}} \leqslant C h^{2}\|f\|_{L^{2}(\Omega)},
$$

with $H^{-1}(\Omega)=H_{0}^{1}(\Omega)^{*}$, holds, where $C$ is independent of $f$ and $h$.

Proof. By definition we have

$$
\left\|u_{2}-u_{2, h}\right\|_{H^{-1}(\Omega)^{2}}=\sup \frac{\left(u_{2}-u_{2, h}, z\right)_{0}}{\|z\|_{H_{0}^{1}(\Omega)^{2}}},
$$

where the supremum has to be taken over all $z \in H_{0}^{1}(\Omega)^{2}$. Let $z$ be fixed. In Raviart-Thomas [12] a linear operator $\Pi_{h}: H^{1}(\Omega)^{2} \rightarrow H_{h}$ has been constructed with

$$
\left\|z-\Pi_{h} z\right\|_{L^{2}(\Omega)^{2}} \leqslant C h\|z\|_{H^{1}(\Omega)^{2}}
$$

and $\operatorname{div} \Pi_{h} z=P_{h} \operatorname{div} z$, where $P_{h}: L^{2}(\Omega) \rightarrow S_{h}$ denotes the $L^{2}(\Omega)$-projection. Using the defining relations of $T$ and $T_{h}$, we therefore get

$$
\begin{aligned}
\left|\left(u_{2}-u_{2, h}, z\right)_{0}\right| & \leqslant\left|\left(u_{2}-u_{2, h}, z-\Pi_{h} z\right)_{0}\right|+\left|\left(u_{1}-u_{1, h}, P_{h} \operatorname{div} z\right)_{0}\right| \\
& \leqslant C\left\{h\left\|u_{2}-u_{2, h}\right\|_{L^{2}(\Omega)^{2}}+\left\|u_{1}-u_{1, h}\right\|_{L^{2}(\Omega)}\right\} \cdot\|z\|_{H^{1}(\Omega)^{2}} .
\end{aligned}
$$

Together with (3.15) the assertion follows.

Using this result, we get

THEOREM 3.2. With the same notations as above, the estimate

$$
\begin{aligned}
&\left\|u_{1}^{(k)}(t)-u_{1, h}^{(k)}(t)\right\|_{L^{2}(\Omega)}+\left\|u_{2}^{(k)}(t)-u_{2, h}^{(k)}(t)\right\|_{H^{-1}(\Omega)^{2}} \\
&+\left|\lambda^{(k)}(t)-\lambda_{h}^{(k)}(t)\right| \leqslant C_{k} h^{2}
\end{aligned}
$$

holds, $C_{k}$ independent of $h$ and $t$.

Proof. We set $H:=L^{2}(\Omega) \times H^{-1}(\Omega)^{2}$ and again apply Theorem 2.2. By (3.15) and (3.20) we get

$$
\left\|\left(T-T_{h}\right) f\right\|_{H} \leqslant C h^{2} .\|f\|_{W}
$$

for $f \in W, W=L^{2}(\Omega)$. The inclusion (2.7) is trivial, and (2.8) is shown in the same way as in the proof of Theorem 3.1; so the assertion follows.

Remark 3.1. If the solution of (3.14) is sufficiently smooth, an estimate analogous to (3.11) can be proved, using $L^{\infty}$-estimates for the mixed finite element method. (See Johnson-Thomée [7], Scholz [14].) 
4. Application II: The Navier-Stokes Problem. In this section we consider the Navier-Stokes equation (stream-function formulation) for an incompressible viscous fluid. Let $\Omega \subset \mathbf{R}^{2}$ be a convex polygonal domain. For $g \in H^{-2}(\Omega)$ we consider the problem

$$
\begin{gathered}
\nu \Delta^{2} \psi+\psi_{x} \Delta \psi_{y}-\psi_{y} \Delta \psi_{x}=g \quad \text { in } \Omega, \\
\psi=\psi_{n}=0 \quad \text { on } \partial \Omega,
\end{gathered}
$$

where $\nu>0$ is the viscosity coefficient; $\Delta \psi$ is the vorticity. (Again we use standard notations of Sobolev spaces; especially, we denote $H^{-k}(\Omega):=H_{0}^{k}(\Omega)^{*}$ and $W^{-k, p}(\Omega):=W_{0}^{k, q}(\Omega)^{*}, k \in \mathbf{N}, 1<p<\infty, 1 / p+1 / q=1$.) A weak formulation of Problem (4.1) is:

Find $\psi \in H_{0}^{2}(\Omega)$ such that

$$
\nu(\Delta \psi, \Delta v)+\left(\psi_{x} \Delta \psi_{y}-\psi_{y} \Delta \psi_{x}, v\right)=(g, v) \text { for all } v \in H_{0}^{2}(\Omega),
$$

where $(\cdot, \cdot)$ denotes the $L^{2}(\Omega)$-scalar product of the pairing between elements of $W^{-k, p}(\Omega)$ and $W_{0}^{k, q}(\Omega)$. (For details we refer to Girault-Raviart [5] or Teman [15], for example.)

For functions $u, v \in C_{0}^{\infty}(\Omega)$ we find, by

$$
u_{x} \Delta u_{y}-u_{y} \Delta u_{x}=\left(u_{x} \Delta u\right)_{y}-\left(u_{y} \Delta u\right)_{x}
$$

and integration by parts, that

$$
\left(u_{x} \Delta u_{y}-u_{y} \Delta u_{x}, v\right)=\left(v_{x} u_{y}-v_{y} u_{x}, \Delta u\right)
$$

this means for $u \in H_{0}^{2}(\Omega)$ we have $u_{x} \Delta u_{y}-u_{y} \Delta u_{x} \in W^{-1,4 / 3}(\Omega)$. Since the operator $T: H^{-2}(\Omega) \rightarrow H_{0}^{2}(\Omega)$, defined by

$$
T f=u \quad \text { if }(\Delta u, \Delta v)=(f, v) \text { for all } v \in H_{0}^{2}(\Omega),
$$

is also continuous from $W^{-1.4 / 3}(\Omega)$ into $W^{3,4 / 3}(\Omega)$ (see Grisvard [6]), the solution of the Navier-Stokes problem fulfills the regularity condition $\psi \in H_{0}^{2}(\Omega) \cap W^{3,4 / 3}(\Omega)$ for $g \in W^{-1,4 / 3}(\Omega)$. If, moreover, $g \in H^{-1}(\Omega)$ holds, the regularity result $\psi \in$ $H_{0}^{2}(\Omega) \cap H^{3}(\Omega)$ follows by Sobolev's imbedding theorem and the fact that $T$ is also continuous as an operator from $H^{-1}(\Omega)$ to $H_{0}^{2}(\Omega) \cap H^{3}(\Omega)$. (Consult also KelloggOsborn [9].)

We want to show that the general theory of Section 2 can be applied if we choose $W:=H^{-2}(\Omega), V:=W^{1,4}(\Omega)$, and $H:=H_{0}^{1}(\Omega)$, and if the right-hand side $g$ of Problem (4.1) is regular enough. The crucial point for further analysis is the following

Lemma 4.1. Let $u, v \in C_{0}^{\infty}(\Omega)$ be given. Then

$$
\left(u_{x} \Delta u_{y}-u_{y} \Delta u_{x}, v\right)=\left(u_{x} u_{y}, v_{y y}-v_{x x}\right)+\left(u_{x}^{2}-u_{y}^{2}, v_{x y}\right)
$$

holds.

Proof. First we use (4.3) and get

$$
\left(u_{x} \Delta u_{y}-u_{y} \Delta u_{x}, v\right)=\left(v_{x} u_{y}-v_{y} u_{x}, \Delta u\right) \text {. }
$$


Now we write

$$
\begin{aligned}
\left(v_{x} u_{y}, \Delta u\right) & =\left(u_{y} u_{x x}+u_{y} u_{y y}, v_{x}\right)=\left(\left(u_{x} u_{y}\right)_{x}-\frac{1}{2} u_{x \mid y}^{2}+\frac{1}{2} u_{y \mid y}^{2}, v_{x}\right) \\
& =-\left(u_{x} u_{y}, v_{x x}\right)+\frac{1}{2}\left(u_{x}^{2}-u_{y}^{2}, v_{x y}\right) .
\end{aligned}
$$

By the same argument we get

$$
\left(v_{y} u_{x}, \Delta u\right)=-\left(u_{x} u_{y}, v_{y y}\right)+\frac{1}{2}\left(u_{y}^{2}-u_{x}^{2}, v_{x y}\right) \text {, }
$$

and the result follows.

Let $g \in H^{-2}(\Omega)$ be fixed. We get $G: V \times \mathbf{R} \rightarrow W$ with

$$
G(u, \lambda):=\lambda\left(u_{x} \Delta u_{y}-u_{y} \Delta u_{x}-g\right) .
$$

Using Lemma 4.1, we see that $G$ is well defined. Further, Problem (4.2) is equivalent to

$$
\psi+T G(\psi, \lambda)=0, \quad \lambda=1 / \nu .
$$

By the regularity results mentioned above, we find $G(\psi, \lambda) \in W^{-1,4 / 3}(\Omega)$ for $g \in W^{-1,4 / 3}(\Omega)$, and $G(\psi, \lambda) \in H^{-1}(\Omega)$ for $g \in H^{-1}(\Omega)$.

Let $V_{h}, 0<h \leqslant h_{0}$, be a family of finite-dimensional subspaces of $H_{0}^{2}(\Omega)$ with the following approximation property:

For $u \in H_{0}^{2}(\Omega) \cap W^{3, q}(\Omega)$ there exists $u_{h} \in V_{h}$ with

$$
\left\|u-u_{h}\right\|_{W^{k . p}(\Omega)} \leqslant C h^{3-k-2(1 / q-1 / p)}\|u\|_{W^{3 . q}(\Omega)},
$$

$0 \leqslant k \leqslant 2,1<q \leqslant p<\infty$, where $C$ is independent of $u$ and $h$.

Remark 4.1. Approximation property (4.6) is typical for conforming finite element subspaces with respect to regular triangulations of $\Omega$ with mesh size $h>0$. (See Ciarlet [3], for example.)

The operator $T_{h}: W \rightarrow V_{h}$ is defined by

$$
T_{h} f=u_{h} \in V_{h} \text { if }\left(\Delta u_{h}, \Delta \varphi\right)=(f, \varphi) \text { for all } \varphi \in V_{h} .
$$

The discrete analogue of Problem (4.5) is

$$
\psi_{h}+T_{h} G\left(\psi_{h}, \lambda\right)=0 .
$$

To apply the general theory of Section 2 we need the following lemmas.

LEMMA 4.2. The estimate

$$
\left\|T-T_{h}\right\|_{\mathscr{L}(W, V)} \leqslant C h^{1 / 2}
$$

holds with $C$ independent of $h$.

Proof. Let $f \in W$ be given. By definition we have

$$
\left\|\left(T-T_{h}\right) f\right\|_{V}=\sup _{z \in W^{-1.4 / 3}(\Omega)} \frac{\left(u-u_{h}, z\right)}{\|z\|_{W^{-1.4 / 3}(\Omega)}} .
$$

For $z \in W^{-1,4 / 3}(\Omega)$ fixed there exists $w \in H_{0}^{2}(\Omega) \cap W^{3,4 / 3}(\Omega)$ such that $\Delta^{2} w=z$ and

$$
\|w\|_{W^{3.4 / 3}(\Omega)} \leqslant C\|z\|_{W^{-1.4 / 3}(\Omega)}
$$

holds with $C$ independent of $z$. We get

$$
\left(u-u_{h}, z\right)=\left(\Delta\left(u-u_{h}\right), \Delta w\right)=\left(\Delta\left(u-u_{h}\right), \Delta(w-\omega)\right), \quad \omega \in V_{h},
$$


where we used the defining equations of $u$ and $u_{h}$, respectively. If $\omega \in V_{h}$ is especially chosen as the orthogonal projection of $w$ onto $V_{h}$ with respect to the scalar product $(\Delta \cdot, \Delta \cdot)$, we get

$$
\left(\Delta\left(u-u_{h}\right), \Delta(w-\omega)\right)=(\Delta u, \Delta(w-\omega)) \leqslant C\|u\|_{H_{0}^{2}(\Omega)} \inf _{\omega \in V_{h}}\|w-\omega\|_{H_{0}^{2}(\Omega)} .
$$

Using the approximation property (4.6) with $k=2, q=4 / 3$, and $p=2$, we find

$$
\inf _{\omega \in V_{h}}\|w-\omega\|_{H_{0}^{2}(\Omega)} \leqslant C h^{1 / 2}\|w\|_{W^{3,4 / 3}(\Omega)} .
$$

By (4.9) and the a priori estimate

$$
\|u\|_{H_{0}^{2}(\Omega)} \leqslant C\|f\|_{H^{-2}(\Omega)}=C\|f\|_{W}
$$

we finally get

$$
\left\|\left(T-T_{h}\right) f\right\|_{V} \leqslant C h^{1 / 2}\|f\|_{W},
$$

and (4.8) is shown.

Remark 4.2. With the same duality arguments, for $f \in H^{-1}(\Omega)$ one can prove

$$
\left\|\left(T-T_{h}\right) f\right\|_{V} \leqslant C h^{3 / 2}\|f\|_{H^{-1}(\Omega)}
$$

and

$$
\left\|\left(T-T_{h}\right) f\right\|_{H} \leqslant C h^{2}\|f\|_{H^{-1}(\Omega)},
$$

$V=W_{0}^{1.4}(\Omega)$ and $H=H_{0}^{1}(\Omega)$.

LEMMA 4.3. Let $\psi$ be a solution of (4.5) and assume $g \in W^{-1,4 / 3}(\Omega)$ : i.e., $\psi \in$ $H_{0}^{2}(\Omega) \cap W^{3,4 / 3}(\Omega)$. For all $u \in H_{0}^{1}(\Omega)$ the estimate

$$
\left\|D_{\psi} G(\psi, \lambda) u\right\|_{W} \leqslant C|\lambda|\|\psi\|_{W^{3.4 / 3}(\Omega)}\|u\|_{H}
$$

holds, $C$ independent of $\psi, \lambda$ and $u$.

Proof. Let $v \in H_{0}^{2}(\Omega)$ be fixed. Using Lemma 4.1 we have

$$
(G(\psi, \lambda), v)=\lambda\left[\left(\psi_{x} \psi_{y}, v_{y y}-v_{x x}\right)+\left(\psi_{x}^{2}-\psi_{y}^{2}, v_{x y}\right)-(g, v)\right],
$$

and, consequently, we find

$$
\left(D_{\psi} G(\psi, \lambda) u, v\right)=\lambda\left(\psi_{x} u_{y}+\psi_{y} u_{x}, v_{y y}-v_{x x}\right)+2 \lambda\left(\psi_{x} u_{x}-\psi_{y} u_{y}, v_{x y}\right) .
$$

We therefore get

$$
\left|\left(D_{\psi} G(\psi, \lambda) u, v\right)\right| \leqslant C|\lambda|\|\psi\|_{W^{1, \infty}(\Omega)}\|v\|_{H_{0}^{2}(\Omega)}\|u\|_{H_{0}^{1}(\Omega)} .
$$

By Sobolev's imbedding theorem we find

$$
\|\psi\|_{W^{1, \infty}(\Omega)} \leqslant C\|\psi\|_{W^{3,4 / 3}(\Omega)}
$$

together with (4.13) we get (4.12).

Now let $\left(\psi_{0}, \lambda_{0}\right)$ be a solution of (4.5) such that (2.3) and (2.6) hold, and let $(\psi(t), \lambda(t))$ and $\left(\psi_{h}(t), \lambda_{h}(t)\right)$ be branches of solutions of (4.5) and (4.7), respectively, according to Theorem 2.1. For simplicity we assume $g \in H^{-1}(\Omega)$, i.e., $\psi(t) \in H_{0}^{2}(\Omega) \cap H^{3}(\Omega)$. Then we get

THEOREM 4.1. For $|t| \leqslant t_{0}, h \leqslant h_{0}$ the estimate

$$
\left\|\psi^{(k)}(t)-\psi_{h}^{(k)}(t)\right\|_{W_{0}^{1.4}(\Omega)}+\left|\lambda^{(k)}(t)-\lambda_{h}^{(k)}(t)\right| \leqslant C_{k} h^{3 / 2},
$$


$k \in \mathbf{N}$, holds. Moreover, for $k=0$,

$$
\left\|\psi(t)-\psi_{h}(t)\right\|_{H_{0}^{1}(\Omega)}+\left|\lambda(t)-\lambda_{h}(t)\right| \leqslant C h^{2}
$$

holds.

Proof. (4.14) is a consequence of Theorem 2.1, Lemma 4.2, and estimate (4.10). Lemma 4.3 shows that Assumption (2.8) of the general theory is fulfilled for $p=2$; (4.15) therefore follows by Theorem 2.2, (4.10), and (4.11).

Remark 4.3. Estimates analogous to (4.14) and (4.15) can be derived if only $g \in W^{-1,4 / 3}(\Omega)$ is assumed.

Département de Mathématiques

École Polytechnique Fédérale de Lausanne

1015 Lausanne, Switzerland

Département de Mathématiques

École Polytechnique Fédérale de Lausanne

1015 Lausanne, Switzerland

Institut für Angewandte Mathematik

Universität Freiburg

78 Freiburg i.Br., Germany

1. H. AMANN, "Fixed point equations and nonlinear eigenvalue problems in ordered Banach spaces," SIAM Rev., v. 18, 1976, pp. 620-709.

2. F. BrezZi, J. RappaZ \& P. A. Raviart, "Finite dimensional approximation of nonlinear problems."

Part I: "Branches of nonsingular solutions," Numer. Math., v. 36, 1980, pp. 1-25; Part II: "Limit points," ibid., v. 37, 1981, pp. 1-28; Part III: "Simple bifurcation points," ibid., v. 38, 1981, pp. 1-30.

3. P. G. Ciarlet, The Finite Element Method for Elliptic Problems, North-Holland, Amsterdam, 1978.

4. J. Descloux \& J. RAPPAZ, "Approximation of solution branches of nonlinear equations," RAIRO Anal. Numér., v. 16, 1982, pp. 319-349.

5. V. GiRault \& P. A. Raviart, Finite Element Approximation of the Navier-Stokes Equations, Lecture Notes in Math., vol. 749, Springer-Verlag, Berlin and New York, 1979.

6. P. Grisvard, Singularité des Solutions du Problème de Stokes dans un Polygone, Séminaires d'Analyse Numérique, Paris, 1978.

7. C. JoHnson \& V. ThOMEE, "Error estimates for some mixed finite element methods for parabolic type problems," RAIRO Anal. Numerr., v. 15, 1981, pp. 41-78.

8. H. B. KelLeR, "Numerical solution of bifurcation and nonlinear eigenvalue problems," Application of Bifurcation Theory (P. H. Rabinowitz, ed.), Academic Press, New York, 1977, pp. 359-384.

9. R. B. KellogG \& J. E. OSBORN, "A regularity result for the Stokes problem in a convex polygon," $J$. Funct. Anal., v. 21, 1976, pp. 397-431.

10. J. NitsCHE, "Ein Kriterium für die Quasi-Optimalität des Ritzschen Verfahrens," Numer Math., v. 11, 1968, pp. 346-348.

11. J. NitsChe, " $L_{\infty}$-convergence of finite element approximations," Mathematical Aspects of Finite Element Methods (Rome, 1975), Lecture Notes in Math., vol. 606, Springer-Verlag, Berlin and New York, 1977, pp. 261-274.

12. P. A. RaviaRt \& J. M. Thomas, "A mixed finite element method for 2-nd order elliptic problems," Mathematical Aspects of Finite Element Methods (Rome, 1975), Lecture Notes in Math., vol. 606, Springer-Verlag, Berlin and New York, 1977, pp. 292-315.

13. R. ScHOLz, " $L_{\infty}$-convergence of saddle-point approximations for second order problems, RAIRO Anal. Numér. v. 11, 1977, pp. 209-216.

14. R. SCHOLz, "Optimal $L_{\infty}$-estimates for a mixed finite element method for second order elliptic and parabolic problems," Calcolo, v. 20, 1983, pp. 355-377.

15. R. Temam, Navier-Stokes equations, North-Holland, Amsterdam, 1977.

16. M. Zlamal, "Curved elements in the finite element method," SIAM J. Numer. Math., v. 10, 1973, pp. $229-240$. 